\title{
¿Cómo evaluaremos la competencia transversal "Planificación y gestión del tiempo" a un alumno de primer curso?
}

L. A. Tortajada Genaro, M. A. Herrero Villén, P. Noguera Murray, S. Morais Ezquerro y J. Atienza Boronat

Departamento de Química. UNIVERSITAT POLITÈCNICA DE VALÈNCIA. Camí de Vera S/N. Valencia (España). 46022. Email: luitorge@qim.upv.es

\begin{abstract}
Learning the competency "Planning and time management" is essential in the current context of university education. Especially interesting is its monitoring in novel freshman since their rates of failure and dropout are often related to mismanagement of available resources, including particularly time planning. The role of instructor is to select the best tools for teaching and assessing the competency, without affecting the course of a particular subject. However, the lack of prior experience in the university community generating uncertainly regarding implementation.

In this paper, a study of the skills associated with this competency combined with a review of published procedures is presented. The context analysis was used to develop a set of guidelines and recommendations of interest to be included during the development of a first-year course. It will also be useful as a support for the acquisition and evaluation of these skills. The potential benefits of the teaching action are to enhance academic performance and, therefore, the satisfaction of students and teachers.
\end{abstract}

Keywords: competency based education, freshman, time management, planning

\footnotetext{
Resumen

El aprendizaje de la competencia "Planificación y gestión del tiempo" es fundamental en el actual marco de la educación universitaria. Es especialmente interesante su evaluación en alumnos noveles dado sus tasas de fracaso y abandono, muchas veces asociadas con una gestión errónea de los recursos disponibles, entre ellos particularmente el tiempo. El papel del profesor es seleccionar las mejores herramientas para la enseñanza y evaluación de esta competencia, sin afectar el transcurso de la asignatura.
}

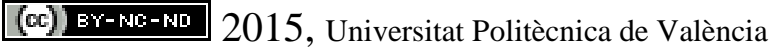

Congreso In-Red (2015): 
¿Cómo evaluaremos la competencia transversal "Planificación y gestión del tiempo" a un alumno de primer curso?

Sin embargo, la falta de claras experiencias previas origina incertidumbres en la comunidad universitaria respecto su implementación.

En esta comunicación, se presenta un estudio de las destrezas asociadas a esta competencia, combinado con una revisión de los procedimientos publicados. El análisis del contexto educativo ha permitido elaborar una serie de pautas y recomendaciones de interés para incorporar durante el desarrollo de una asignatura de primer curso. También servirá como material de apoyo para la adquisición y la evaluación de estas destrezas. Los beneficios potenciales de la acción docente son mejorar los rendimientos académicos $y$, por tanto, la satisfacción de los alumnos y los profesores.

Palabras clave: educación basada en competencias, primer curso, gestión del tiempo, planificación

\section{Introducción}

La Declaración de Bolonia, publicada en 1999, introdujo un gran cambio en la educación superior, centrándola en el alumno y en un aprendizaje activo del mismo. Sin embargo, desde entonces, el marco educativo de la educación superior está sufriendo una serie de cambios que pretenden conseguir que los alumnos adquieran competencias para afrontar los retos del nuevo milenio. Este cambio de paradigma está provocando que la educación superior se centre en el desarrollo de competencias y resultados de aprendizaje. Se pretende, de este modo, que los resultados de los programas universitarios estén constituidos por la adquisición de competencias específicas de área (fundamentalmente) y por la adquisición de una serie de competencias genéricas (habilidades transferibles) (Wagenaar, 2014).

La selección de las competencias genéricas que una institución universitaria desea que posean sus futuros egresados no debe realizarse a la ligera, ya que en el mundo laboral está aceptado que la empleabilidad y la cualificación de los candidatos depende en gran medida de las competencias personales o genéricas que éstos pueden aportar para el desempeño de sus tareas (pudiendo, incluso, ser de mayor interés que los propios conocimientos técnicos) (García et al., 2008). Es fácil encontrar listados de competencias genéricas de mayor o menor extensión (Lokhoff et al, 2010; García et al. 2008). Para decidir y seleccionar el perfil competencial a adquirir por los alumnos, debe considerarse normativas, directrices y experiencias previas a nivel estatal y europeo, sino que, además, hay que conseguir que las competencias seleccionadas garanticen el marco de referencia de algunas titulaciones con regulaciones o recomendaciones específicas. La Universitat Politècnica de València (UPV) inició el Proyecto de Competencias Transversales en el curso 2013-2014, que pretendía ser

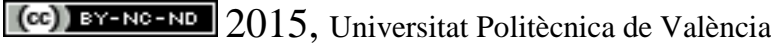


el punto de inflexión en la formación basada en competencias. Fruto de este Proyecto, se definieron una serie de 13 competencias, que en la UPV se denominaron competencias transversales (Tabla 1). Además, para cada una de ellas, la UPV establece diferentes niveles de dominio o resultados de aprendizaje, correspondientes al Grado (dos niveles) y Máster (uno único), y que indican los aprendizajes que los estudiantes deben alcanzar al finalizar cada uno de dichos niveles.

\section{Tabla 1. Competencias transversales de la Universitat Politécnica de Valencia}

Comprensión e integración

Aplicación y pensamiento práctico

Análisis y resolución de problemas

Innovación, creatividad y emprendimiento

Diseño y proyecto

Conocimiento de problemas contemporáneos

Responsabilidad ética, medioambiental y profesional
Comunicación efectiva

Pensamiento crítico

Trabajo en equipo y liderazgo

Aprendizaje permanente

Planificación y gestión del tiempo

Instrumental específica

Este trabajo presenta una propuesta que describe el modo de adquirir y evaluar la competencia transversal "Planificación y gestión del tiempo" a un alumno de primer curso.

La planificación y gestión del tiempo es una habilidad crucial en la vida profesional, particularmente, tras la expansión mundial del concepto competitividad (Claessens, 2007). En este sentido, se han publicado diferentes estrategias centradas en la forma en que las personas en distintas organizaciones administran su tiempo, y cómo estos esfuerzos se pueden mejorar (Mackenzie, 2009). Por otra parte, la gestión del tiempo es un tema importante en educación, puesto que los estudiantes deben utilizarlo para cumplir con sus responsabilidades académicas (Mirzaei, 2012). Una queja general de los estudiantes es que hay muchas clases, actividades o exámenes que requieren su constante dedicación, originando consecuencias negativas, como ansiedad, baja calidad y peor rendimiento. Por lo tanto, la educación universitaria debe incluir la formación en esta competencia para mejorar tanto el desarrollo de la vida universitaria como la preparación para la vida laboral.

La "gestión del tiempo" es en realidad un término engañoso, porque es un factor inaccesible; sería más apropiado hablar de autogestión en relación con el desempeño de una o múltiples tareas dentro de un cierto período de tiempo. Así, la gestión del tiempo implica el establecimiento de metas, cumplimiento de plazos, adaptación frente al cambio, hacer planes y organizar eficazmente su tiempo (Nadinloyi, 2013). Algunos de sus beneficios incluyen la reducción de la dilación, tener más control, menos estrés, completar más tareas y, generalmente, con mayor éxito (Eilam y Aharon, 2003). El establecimiento de prioridades y la evaluación de su progreso permiten asegurar la asignación del tiempo suficiente en las diferentes actividades académicas.

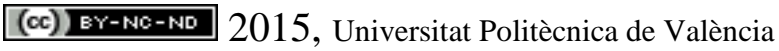

Congreso In-Red (2015): 
¿Cómo evaluaremos la competencia transversal "Planificación y gestión del tiempo" a un alumno de primer curso?

Esta competencia, al ser un conjunto de hábitos o comportamientos, puede ser adquirida a través de una mejor formación (McCann, 2012). La gestión del tiempo puede ser influenciada por la cognición (por ejemplo, la fijación de objetivos y la intención) y el contexto (por ejemplo, papel del ambiente de estudio).

A pesar de la relevancia de la competencia "Planificación y gestión del tiempo", en general, son pocas las actividades que refuerzan el uso del tiempo con eficacia, más allá de completar un trabajo, práctica o examen dentro de los plazos. Esto se complica cuando se considera que el resultado al aplicar una estrategia varía de acuerdo a las capacidades y limitaciones de cada persona (Langa, 2013). Además, tal como indican los estudios empíricos existentes sobre este tipo de competencias en las clases universitarias, su evaluación exige una metodología específica (Allen, 2005; Xu, 2013). Por lo tanto, para llevar al aula realmente este reto de forma masiva como exige el nuevo panorama educativo universitario, se debe reflexionar críticamente sobre cómo incorporar los conceptos y los métodos asociados a esta competencia transversal sin alterar el correcto funcionamiento de la dinámica de cada asignatura.

\section{Objetivos}

El principal objetivo de la presente investigación es realizar un estudio de las herramientas disponibles para la adquisición y evaluación de la competencia "Planificación y gestión del tiempo" mediante estrategias que sean efectivas, eficaces y flexibles. Los objetivos específicos son:

- Análisis de la situación actual.

- Análisis de las destrezas requeridas para una gestión del tiempo eficaz

- Análisis de las herramientas de evaluación de la competencia

- Diseño de acciones futuras adaptadas al alumno novel

Con estas herramientas y en el marco de la docencia de primer curso de grado, se pretende constatar que los alumnos son capaces de planificar adecuadamente el tiempo disponible, programando las actividades de forma que les permitan alcanzar dicho fin.

\section{Desarrollo de la innovación}

La investigación se ha centrado en el estudio de las metodologías aplicables a la enseñanza y evaluación de la competencia transversal "Planificación y gestión del tiempo”. Para ello, se ha tenido en cuenta las directrices que la propia UPV ha marcado para la evaluación de las trece competencias transversales que ha definido. En este programa, se ha establecido

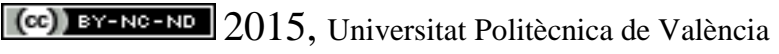


que las competencias se adquieran progresivamente a lo largo de tres niveles de dominio, que se corresponden con el Grado (dos niveles) y con el Máster (un único nivel) (Tabla 2). Hay que resaltar que estos resultados de aprendizaje son orientativos tanto para los profesores como para los estudiantes.

La investigación se ha centrado en las primeras etapas del nivel de Grado identificándose las destrezas o habilidades requeridas para una gestión del tiempo efectiva para un alumno de primer curso. Además, se ha realizado un análisis de los procedimientos y herramientas para la evaluación, de acuerdo con los estudios publicados en relación con la planificación y gestión del tiempo.

\section{Tabla 2. Aspectos establecidos en la Universitat Politècnica de València para la competencia} "Planificación y gestión del tiempo"

\section{Definición}

Planificar adecuadamente el tiempo disponible y programar las actividades necesarias para alcanzar los objetivos, tanto académico-profesionales como personales.

\section{Descripción}

Esta competencia tiene relación con el establecimiento de objetivos, con la planificación y programación de actividades (tiempo y fases) y con la organización y gestión de los recursos necesarios para alcanzar objetivos.

En prácticamente todas las definiciones de Organización y Planificación es posible hallar elementos comunes importantes: Establecer objetivos; Elección de los medios más convenientes para alcanzarlos antes de emprender la acción (planes y programas); Toma de decisiones previa a la acción; y, Decidir con anticipación lo que hay que hacer y cómo deberá hacerse.

Resultados de aprendizaje para el grado ( 2 niveles)

- Definir claramente los objetivos a cumplir a corto y medio plazo

- Jerarquizar los objetivos según criterios de importancia

- Planificar las actividades a desarrollar a corto y medio plazo

- Cumplir la planificación

- Evaluar los resultados alcanzados

Resultados de aprendizaje para el máster (un único nivel)

- Definir claramente los objetivos a alcanzar en el tiempo colectivo (grupos de trabajo, reuniones, entrevistas...)

- Establecer prioridades en los objetivos integrando las individuales y las grupales.

- Planificar las actividades a desarrollar en un proyecto complejo

- Organizar los procesos y procedimientos adecuados al proyecto:

- Elaborar los proyectos con una lógica apropiada a las tareas que le incumben

- Planificar con una clara conciencia de los medios y tiempos disponibles

- Planificar previendo el control de las actividades para conseguir los resultados

- Cumplir la planificación comprobando que el tiempo colectivo se utiliza

- Evaluar los resultados alcanzados tanto individual como grupalmente 
¿Cómo evaluaremos la competencia transversal "Planificación y gestión del tiempo" a un alumno de primer curso?

\section{Resultados}

\subsection{Análisis de la situación actual}

El estudio ha indicado qué tres grandes temas limitan el planteamiento básico del problema a abordar, a pesar de que aparece definida como una de las competencias transversales que los alumnos invariablemente deben alcanzar:

a) Nuevo escenario educativo. Los alumnos universitarios analizados se enfrentan a un nuevo paradigma del aprendizaje cuando se matriculan en la educación superior. La formación basada en competencias es el eje donde se construye la preparación de su futuro profesional. Entre ellas, las competencias transversales sintetizan el perfil que deben adquirir todos los alumnos y cubren el marco de referencia de algunas titulaciones.

b) Estudiantes noveles. La enseñanza de esta competencia es especialmente crítica en los alumnos de nuevo ingreso debido a los cambios que experimentan, incluyendo patrones de aprendizaje, relaciones con compañeros y demandas académicas. Las habilidades relacionadas con la gestión del tiempo son fundamentales para facilitar esta transición y minimizar las tasas de fracaso y abandono.

c) Profesores "noveles". La incorporación del aprendizaje basado en competencias en la formación de los estudiantes exige también diseñar e implementar procesos de aprendizaje y evaluación de dichas competencias a lo largo de la vida académica universitaria. Sin embargo, existe poca experiencia en la comunidad universitaria (profesores, gestores e instituciones de apoyo). A parte de los documentos marco, no se proporciona información de interés que apoye la puesta a punto en el aula. De hecho, la formación de los profesores en este ámbito se está produciendo simultáneamente a la implementación de los programas. Esto lamentablemente conlleva a sistemas basados en experiencias de prueba y error, que pueden traducirse en promociones de alumnos que sufren las incertidumbres asociadas a estos cambios.

\subsection{Análisis de las destrezas requeridas}

De forma genérica, la identificación de las habilidades requeridas determina los resultados de aprendizaje para el nivel de la competencia a alcanzar. En el caso del aprendizaje de esta competencia, una gestión del tiempo eficaz y efectiva exige la fijación de objetivos, con la planificación y programación de actividades (tiempo y fases) y con la organización y gestión de los recursos necesarios para alcanzar objetivos (Fig.1). De acuerdo con las directrices marcadas por la UPV y diferentes autores (García-Ros, 2004; Nadinloyi, 2013), los hitos a cumplir a corto y medio plazo para adquirir la competencia por un estudiante de grado son jerarquizar los objetivos de aprendizaje según criterios de importancia, planificar

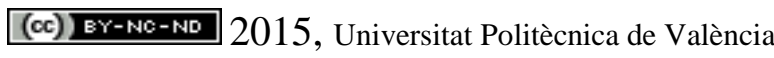


las actividades a desarrollar a corto y medio plazo, cumplir la planificación y evaluar los resultados alcanzados de acuerdo con las directrices marcadas (Tabla 2). Sin embargo, la dificultad está en llevar a cabo dicha idea en el aula universitaria. Algunas de las habilidades requeridas están cubiertas con actividades formativas que conllevan la planificación de la acción, incluyendo el establecimiento de metas claras, dividiendo la tarea en pasos discretos, y la revisión de su progreso hacia la meta. Otras habilidades exigen actividades formativas específicas para fomentar la priorización y la organización. En esta categoría se encuentra habilidades como centrarse en tareas urgentes e importantes, más que en las que no son importantes o no te mueven hacia las metas, elaborar lista de decisiones, evitar la dilación o perseverar cuando las cosas no están funcionando. De este modo, se le proporciona al alumnado la libertad de dirigir su aprendizaje, en el marco de la asignatura, pero de acuerdo a sus propios intereses y a los objetivos o resultados de aprendizaje a alcanzar para esta competencia.

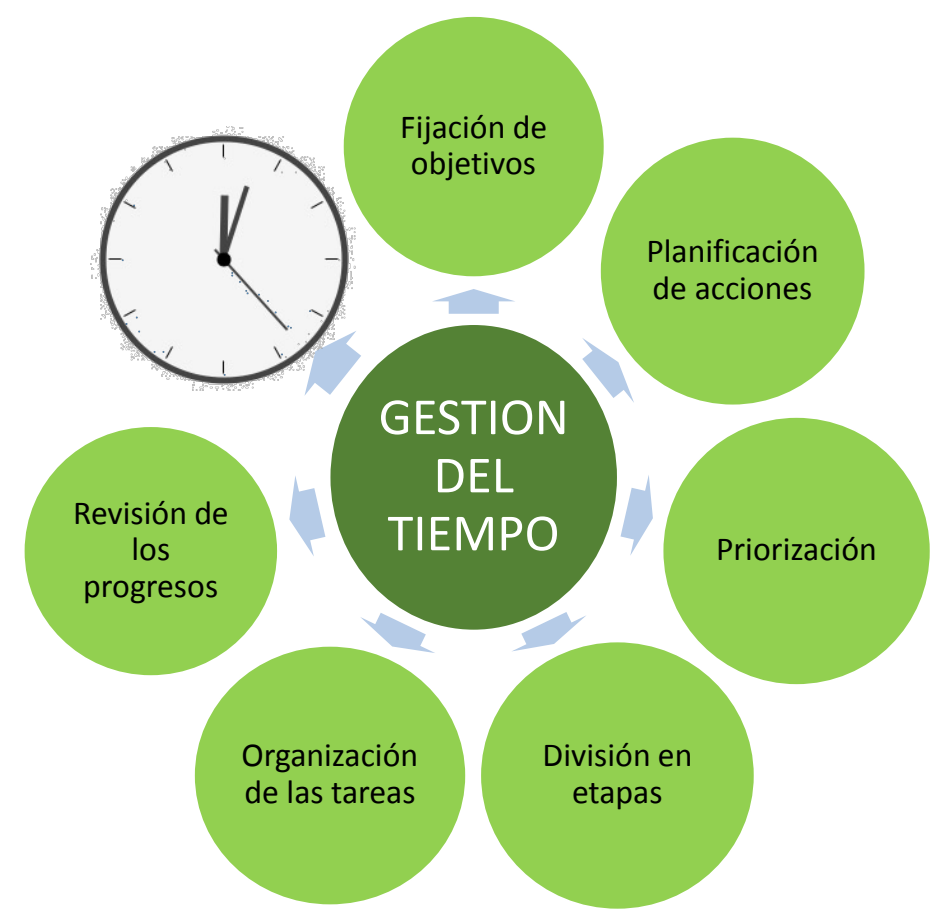

Fig. 1 Esquema de los principales hitos asociados a la competencia "Planificación y Gestión del tiempo" 
¿Cómo evaluaremos la competencia transversal "Planificación y gestión del tiempo" a un alumno de primer curso?

\subsection{Recursos de trabajo y evaluación}

Las actividades formativas propuestas deben ser principalmente genéricas, dado que la gestión del tiempo es aplicable prácticamente a cualquier actividad. De este modo, se mantiene el aprendizaje de las competencias específicas de la materia que se está impartiendo, pero se refuerza la planificación de la acción y se fomenta la priorización y la organización.

Dada la repercusión que posee la gestión del tiempo, tanto en el ámbito académico como el profesional o el personal, el número de recursos para adquirir y evaluar las destrezas asociadas con esta competencia es elevado (Tabla 3). Dado que el presente trabajo explora la adquisición y evaluación de esta competencia al nivel más elemental (primer curso de grado, alumno novel) para la evaluación de la misma, de entre las posibles herramientas, se han seleccionado: la autoevaluación, la realización de diagramas de flujo y los informes de prácticas de laboratorio.

Tabla 3. Algunos recursos para adquirir y evaluar destrezas asociadas a la "Planificación y Gestión del tiempo"

Genéricas
Trabajos académicos
Resolución problemas
Informes prácticas laboratorio
Informes prácticas externas

\section{Genéricas}

Trabajos académicos

Informes prácticas externas

\section{Específicas}

Autoevaluación

Organigramas

Diagramas de Gantt

Otros sistemas de gestión
Globales

Proyectos

Contrato de aprendizaje

Casos

Portafolios

\subsubsection{Auto-evaluación}

Entre las diferentes opciones descritas, la autoevaluación parece la mejor estrategia para afrontar la diversidad de intereses, necesidades y ritmos de aprendizaje del alumnado respecto esta competencia transversal. El principal inconveniente es que es una herramienta subjetiva porque es el propio alumno el que se evalúa a sí mismo y es difícil establecer una valoración cuantitativa del grado de adquisición de la competencia. No obstante, la autoevaluación tiene una ventaja asociada: es la estrategia por excelencia para aprender a valorar, criticar y a reflexionar sobre el proceso de enseñanza y aprendizaje individual realizado. En el área de la autoevaluación, existen herramientas como diarios, portafolios, cuestionarios, listas de control, tablas, escalas de estimación, entre otras para recoger las informaciones relevantes.

Para el tipo de alumno a los que se pretende evaluar y el nivel de adquisición de la competencia, se ha seleccionado la autoevaluación basada en cuestionarios (Claessens, 2007). Se trata de una actividad en la que el estudiante evidencia cómo afronta la planificación y gestión de su tiempo formativo y permite valorar la relación entre los

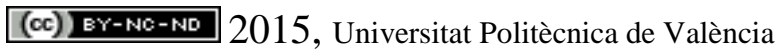


esfuerzos realizados y el rendimiento académico conseguido. A diferencia del resto de herramientas disponibles (Tabla 3), la evaluación está muy dirigida por el profesor pero únicamente proporciona datos sobre los procesos de enseñanza y aprendizaje. Sin embargo, se puede diseñar de forma que el alumno sea consciente de su capacidad para organizarse y los diferentes ritmos de estudio o trabajo que aplica. De este modo, el alumno conoce y toma conciencia de cuál es su progreso, a la vez que desarrolla la capacidad de autogestión y posibilita que pueda repetirlo en el tiempo de forma autónoma. Otros aspectos positivos de los cuestionarios de auto-evaluación son su carácter motivador y de refuerzo del aprendizaje.

Al comparar los distintos cuestionarios de autoevaluación disponibles en la bibliografía que evalúan específicamente esta competencia (aplicados al contexto universitario), se observa que todos posibilitan que los alumnos valoren su propio aprendizaje de la competencia y fomentan la autonomía del alumno (Bond, 1988; Britton, 1991 y Macan, 1990). Sin embargo, los cuestionarios analizados poseen un elevado número de preguntas (más de 30), lo que limita su implementación rutinaria durante el desarrollo de una asignatura. Por lo tanto, se ha puesto de manifiesto la necesidad de introducir mejoras o innovaciones. A la hora de diseñar un cuestionario se pueden los bloques de preguntas indicados en la Tabla 4.

Tabla 4. Diseño de la autoevaluación basada en un cuestionario

\begin{tabular}{ll}
\hline Bloque & Contenido \\
\hline I & Percepción del control sobre el tiempo \\
II & Establecimiento de metas \\
III & Estructuración del tiempo \\
IV & Efectividad en la organización \\
V & Persistencia \\
VI & Consecución de objetivos \\
\hline
\end{tabular}

Otra posible herramienta a utilizar es la evaluación mediante rúbricas, sin embargo, se considera que al alumno novel le es difícil distinguir, o apreciar, los diferentes niveles de adquisición de los resultados de su aprendizaje. Es preferible que un alumno rellene a lo largo de un semestre varios cuestionarios de respuesta múltiple que rúbricas, ya que completar estas últimas le puede resultar una tarea ardua.

No obstante, es posible realizar una evaluación por parte del profesor mediante rúbricas en las actividades presenciales, especialmente de las sesiones de prácticas. Sin embargo, los autores consideran esto una tarea difícil, dado que los grupos de primero son numerosos, por lo que a priori se descarta el uso de esta herramienta.

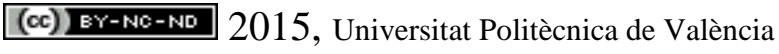

Congreso In-Red (2015): 
¿Cómo evaluaremos la competencia transversal "Planificación y gestión del tiempo" a un alumno de primer curso?

\subsubsection{Diagramas de flujo}

Un diagrama de flujo es una representación abreviada de una experiencia o de un procedimiento. En el caso de procesos industriales (o programación), los diagramas de flujo tienen que seguir unas pautas concretas, incluso existe normativa a la hora de su realización. Sin embargo, los diagramas de flujo utilizados para esquematizar procesos en los laboratorios de química tienen una gran componente personal, adaptándolos el usuario a sus necesidades y requerimientos. Los diagramas de flujo deben tener un inicio (y un final) claro, que suele ser un reactivo (o un producto), y los procesos en los que se añade (o separan) componentes se indican mediante flechas (Fig. 2). Una ventaja adicional de los diagramas de flujo es que se pueden anotar observaciones a medida que se realiza el experimento.

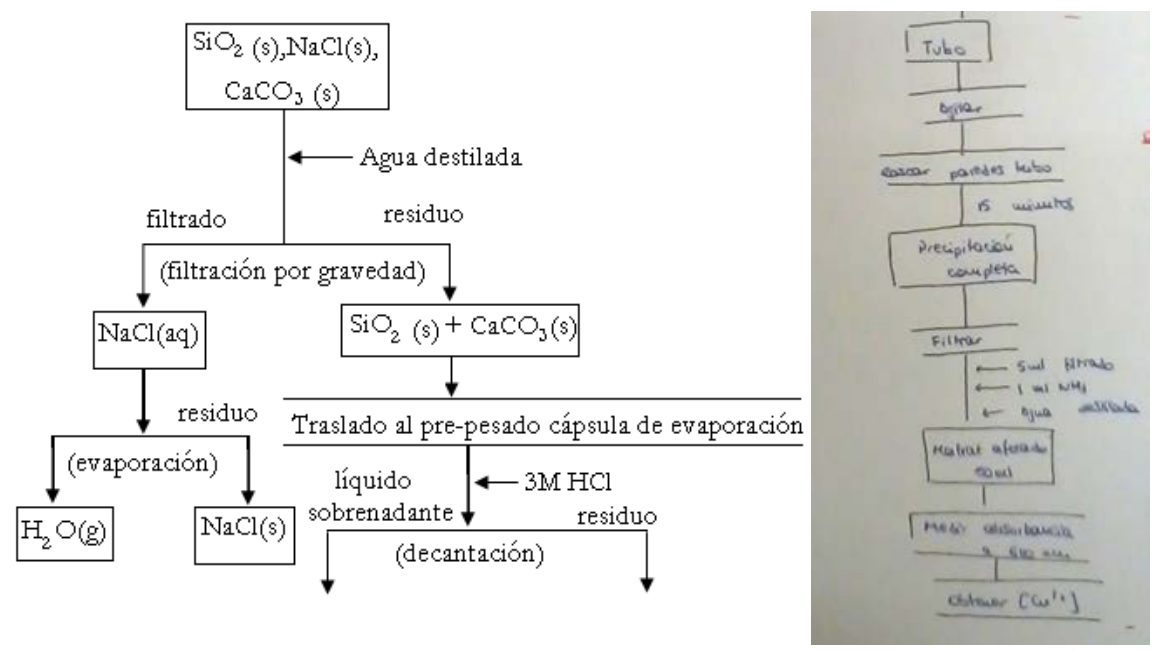

Fig. 2. Ejemplos de algunos diagramas de flujo

La principal ventaja que presenta un diagrama de flujo, independientemente del proceso al que se aplica, es que favorecen la comprensión del mismo, al mostrarlo como un dibujo donde las distintas etapas están claramente marcadas. De este modo, un buen diagrama de flujo puede reemplazar varias páginas de texto. La utilización de esta herramienta en la docencia permite al alumno identificar qué puntos tiene claros o no. Además, al ser una herramienta personal, el alumno lo adapta a su método de aprendizaje.

Por otro lado, la evaluación de la idoneidad, o no, del diagrama de flujo realizado por un alumno en el primer curso de grado es muy sencilla, especialmente cuando en dicho diagrama de flujo se planifica una sesión o práctica de laboratorio. Tras la experiencia en los últimos cuatro años por parte de los autores, se considera necesario generar unas

(cc)) EY-Nc-ND 2015, Universitat Politècnica de València 
instrucciones claras o pautas para que el alumno aprenda a utilizar y manejar este tipo de diagramas. Por ello, es indispensable la inclusión de estas instrucciones en el propio libro de prácticas.

\subsubsection{Informes de prácticas de laboratorio}

Los informes de prácticas pueden incluir la planificación del trabajo a realizar, para ello será necesario que los alumnos definan los objetivos parciales a cumplir. Se podría solicitar al alumno la inclusión de dos diagramas de flujo, el inicial y el finalmente aplicado, indicando el tiempo dedicado a cada una de las tareas, para así poder verificar si la planificación ha cumplido las expectativas.

\subsubsection{Otras herramientas de ayuda al alumno}

Se puede proponer a los alumnos que registren cómo gestionan el tiempo, estableciendo objetivos, listando tareas y metas y fijando los plazos. La clave también es que los alumnos aprendan a priorizar, recomendado una planificación diaria, semanal y cuatrimestral. Para ello, disponen de herramientas o estrategias específicas que se aplican diariamente en la vida profesional para discriminar en función de la urgencia e importancia de la tarea (análisis ABC, el análisis Pareto, método de Eisenhower, método de la reacción domino, método POSEC, ...).

Por otro lado, no hay que olvidar que el Instituto de Ciencias de la Educación también contribuye a la formación integral de los estudiantes, impartiendo cursos para la adquisición de competencias genéricas. De hecho, dentro de su programa de asesoramiento al aprendizaje tiene un curso denominado "Gestión eficaz del tiempo para mejorar tu rendimiento" donde los alumnos aprenden técnicas para planificar y gestionar el tiempo.

Además, se resaltará que también existen recursos disponibles que facilitan la planificación, ejecución y evaluación de las tareas. En esta categoría se encuentra sistemas en soporte físico (agendas, calendarios, etc.) y en soporte digital (agenda de PoliformaT, programas para ordenadores, aplicaciones para móviles, etc.).

\subsection{Diseño de acciones}

Para implementar correctamente la enseñanza de esta competencia, se ha diseñado una acción, cuyo esquema temporal se muestra en la Fig. 3. Se han priorizado las metas a lograr y las herramientas a utilizar de acuerdo a los análisis anteriores. Inicialmente, el profesor debe fijar los objetivos de aprendizaje y criterios de evaluación asociados a esta competencia e informar al alumno de los mismos. Para el desarrollo, cualquier actividad enseñanza-aprendizaje podría adaptarse para trabajar esta competencia transversal. Nuestra propuesta implica que la adquisición de la competencia se realice principalmente en las 
¿Cómo evaluaremos la competencia transversal "Planificación y gestión del tiempo” a un alumno de primer curso?

sesiones de prácticas. Asimismo, las tutorías pueden actuar como escenario de apoyo para aquellos alumnos que posean más problemas o quieran reforzar esta competencia.

Respecto a la evaluación, los cuestionarios adaptados y los diagramas de flujo parecen ser instrumentos fiables para evaluar cómo los estudiantes gestionan su tiempo, e incluso pueden servir para predecir su rendimiento en el primer año universitario. Asimismo, se propone la inclusión de estrategias de retro-alimentación para que, en función de los resultados, los alumnos puedan conocer las soluciones específicas para cada una de las deficiencias detectadas.

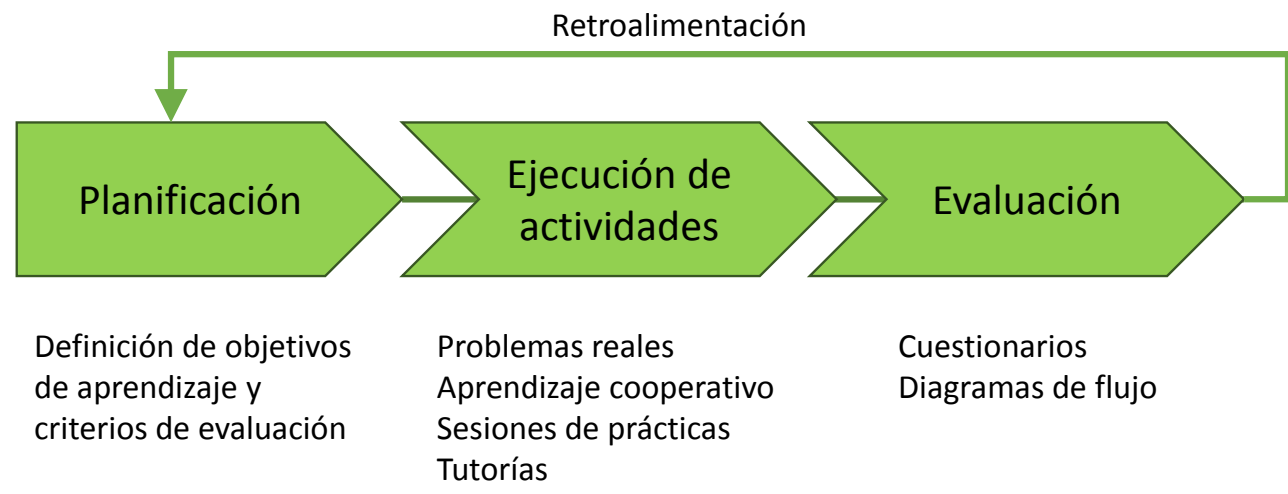

Fig. 3. Esquema de las acciones para la enseñanza y evaluación de la competencia "Planificación y Gestión del tiempo"

\section{Conclusiones}

Una competencia transversal como la gestión del tiempo es esencial en el ámbito laboral para transformar un conocimiento en comportamiento, y por lo tanto, la educación universitaria tiene que garantizar su adquisición. A pesar de existir diferentes herramientas de apoyo a la planificación y gestión del tiempo, la comunidad universitaria no está acostumbrada, en general, a la enseñanza ni a la evaluación de esta competencia. Es necesario crear un nuevo marco de acción que permita llevar este objetivo a la realidad del aula, registrando evidencias de su grado de adquisición. Tras el análisis de las distintas opciones disponibles, las herramientas recomendadas parecen indicar que se alcanzará esta meta sin poner en peligro el aprendizaje de las competencias más específicas propias de la titulación y de la asignatura. Particularmente, el uso de cuestionarios y diagramas de flujo permitirá, de forma fiable, establecer una valoración de su grado de adquisición, tanto para el propio alumno como para el profesor, predecir los resultados académicos y desarrollar estrategias para mejorar las destrezas relacionadas con esta competencia.

(cc)) EY-Nc-ND 2015, Universitat Politècnica de València 
No obstante, los especialistas en psicología y pedagogía son los verdaderos responsables en la formación y evaluación de las competencias transversales centradas en el comportamiento. Por ello, en lugar de transferir esta responsabilidad a los docentes de asignaturas específicas de cada titulación, se recomendaría que los Servicios Universitarios de apoyo a la docencia o gabinetes psicopedagógicos ampliasen la oferta y duración de los cursos específicos destinados a los alumnos, siendo los responsables la formación y evaluación de las competencias transversales con garantías.

\section{Referencias}

ALLEN, J., RAMAEKERS, G. y VAN DER VELDEN, R. (2005). "Measuring competencies of higher education graduates" en New Directions for Institutional Research, 126, 49-59.

BOND, M. J. y FEATHER, N. T. (1988). "Some correlates of structure and purpose in the use of time” en Journal of Personality and Social Psychology, 55(2), 321.

BRITTON, B. K. y TESSER, A. (1991). "Effects of time-management practices on college grades” en Journal of Educational Psychology, 83(3), 405.

CLAESSENS, B. J., VAN EERDE, W., RUTTE, C. G., y ROE, R. A. (2007). “A review of the time management literatura" en Personnel Review, 36(2), 255-276.

EILAM, B. y AHARON, I. (2003). "Students' planning in the process of self-regulated learning” en Contemporary Educational Psychology, 28(3), 304-334.

GARCÍA GARCÍA, M. J., FERNÁNDEZ SANZ, L., TERRÓN LÓPEZ, M. J. y BLANCO ARCHILLA, Y. (2008). Métodos de evaluación para las competencias genéricas más demandadas en el mercado laboral. (AENUI, Ed.) en Actas de las XIV Jornadas de Enseñanza universitaria de la Informática (JENUI 2008), pp. 265-272.

GARCÍA-ROS, R., PÉREZ-GONZÁLEZ, F. y HINOJOSA, E. (2004). “Assessing Time Management Skills as an Important Aspect of Student Learning The Construction and Evaluation of a Time Management Scale with Spanish High School Students” en School Psychology International, 25(2), 167-183.

LANGA, C. (2013). "Management of Time Resources for Learning through Individual Study in Higher Education” en Procedia-Social and Behavioral Sciences, 76, 13-18.

LOKHOFF, J., WEGEWIJS, B., DURKIN, K, WAGENAAR, R., GONZÁLEZ, J., ISAACS, A.K., DONÁ DALLE ROSE, L.F. y GOBBI, M. (Eds.) (2010). A Tuning guide to formulating degree programme profiles: Including programme competences and programme learning outcomes. Universidad de Deusto (Bilbao), 64 pp.

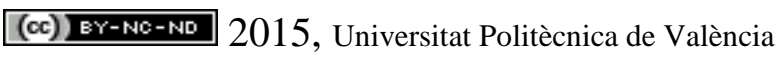

Congreso In-Red (2015): 
¿Cómo evaluaremos la competencia transversal "Planificación y gestión del tiempo" a un alumno de primer curso?

MACAN, T. H. (1994). “Time management: Test of a process model” en Journal of Applied Psychology, 79(3), 381.

MACCANN, C., FOGARTY, G. J. y ROBERTS, R. D. (2012). "Strategies for success in education: Time management is more important for part-time than full-time community college students” en Learning and Individual Differences, 22(5), 618-623.

MACKENZIE, A. y NICKERSON, P. (2009). "The time trap: The classic book on time management” AMACOM Div American Mgmt Assn. New York

MIRZAEI, T., OSKOUIE, F. y RAFII, F. (2012). "Nursing students' time management, reducing stress and gaining satisfaction: a grounded theory study” en Nursing \& Health Sciences, 14(1), 46-51.

NADINLOYI, K. B., HAJLOO, N., GARAMALEKI, N. S. y SADEGHI, H. (2013). “The study efficacy of time management training on increase academic time management of students” en Procedia-Social and Behavioral Sciences, 84, 134-138.

WAGENAAR, R. (2014) Competences and learning outcomes: a panacea for understanding the (new) role of Higher Education? en Tuning Journal for Higher Education, 1(2), 279-302

XU, J., DU, J. y FAN, X. (2013). "Finding our time: Predicting students' time management in online collaborative groupwork” en Computers \& Education, 69, 139-147.

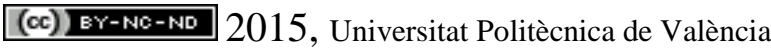
Congreso IN-RED (2015): 